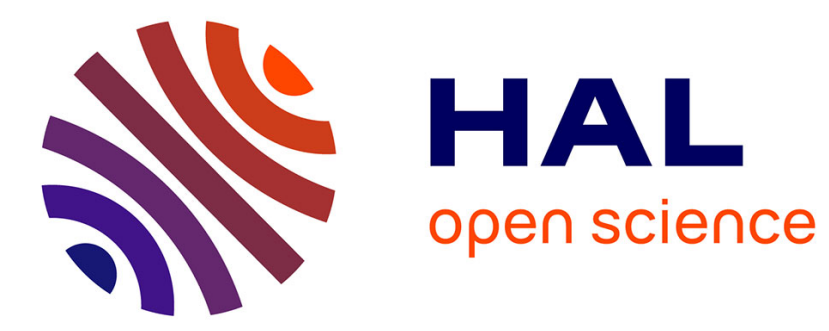

\title{
Interprétation d'expériences sur des solutions diluées de polymère en milieu poreux
}

\author{
S. Daoudi
}

\section{To cite this version:}

S. Daoudi. Interprétation d'expériences sur des solutions diluées de polymère en milieu poreux. Journal de Physique Lettres, 1976, 37 (3), pp.41-42. 10.1051/jphyslet:0197600370304100 . jpa-00231230

\section{HAL Id: jpa-00231230 https://hal.science/jpa-00231230}

Submitted on 1 Jan 1976

HAL is a multi-disciplinary open access archive for the deposit and dissemination of scientific research documents, whether they are published or not. The documents may come from teaching and research institutions in France or abroad, or from public or private research centers.
L'archive ouverte pluridisciplinaire HAL, est destinée au dépôt et à la diffusion de documents scientifiques de niveau recherche, publiés ou non, émanant des établissements d'enseignement et de recherche français ou étrangers, des laboratoires publics ou privés. 


\title{
INTERPRÉTATION D'EXPÉRIENCES SUR DES SOLUTIONS DILUÉES DE POLYMÈRE EN MILIEU POREUX
}

\author{
S. DAOUDI \\ Physique de la Matière condensée, Collège de France \\ 11, Place Marcelin-Berthelot, 75231 Paris Cedex 05, France
}

(Reçu le 2 décembre 1975, accepté le 6 janvier 1976)

\begin{abstract}
Résumé. - Des expériences récentes d'écoulement de solutions diluées de polymère en milieu poreux montrent un accroissement très grand de la perte de charge de la solution par rapport au solvant à partir d'une certaine vitesse, mais l'écart s'atténue pour des vitesses beaucoup plus élevées. Une interprétation qualitative de ces résultats est proposée, basée sur un modèle moléculaire simple de la transition coil-stretch.
\end{abstract}

Abstract. - Recent experiments on the flow of dilute polymer solutions through porous media indicate a very large increase of the solution head loss relative to the solvent above a certain velocity, but the deviation decreases at much higher velocities. A qualitative interpretation of these results is proposed based on a simple molecular model of the coil-stretch transition.

L'étude de l'écoulement d'une solution de polymère à travers un milieu poreux présente des difficultés tant sur le plan expérimental que théorique. En plus des problèmes liés au polymère (adsorption, rétention, agglomération, dégradation, ...), la géométrie de l'écoulement est compliquée et mal connue. Mais l'intérêt pratique des solutions de polymère dans la récupération du pétrole a stimulé beaucoup de recherches sur ce sujet.

Dans un article récent, [1] James et Mac Laren exposent des expériences intéressantes d'écoulement de solutions aqueuses diluées de Poe dans un milieu poreux constitué par un empilement de petites billes sphériques. La taille des billes est telle que les effets d'adsorption et de rétention soient négligeables. Ils mesurent la chute de pression $\Delta p$ en fonction de la vitesse de débit $V$ en faisant varier la masse moléculaire $M$ du polymère, sa concentration $c$ et le diamètre $D$ des billes. La figure 1 qui est une reproduction partielle de leur figure 13, représente la perte de charge par unité de longueur $\Delta p / \rho g L$ ( $\Delta p$ est mesuré sur une longueur $L, \rho$ étant la masse volumique de la solution et $g$ l'accélération de la pesanteur) en fonction de la vitesse pour 3 valeurs de la concentration. En suivant le sens des vitesses croissantes, on observe un écart des solutions par rapport au comportement newtonien du solvant qui débute à une certaine valeur de $V$, croît brusquement jusqu'à une valeur relative très élevée puis retombe progressivement à zéro. Pour les deux premières valeurs de $c$, le système est instable dans un certain intervalle de vitesses (courbes interrompues).

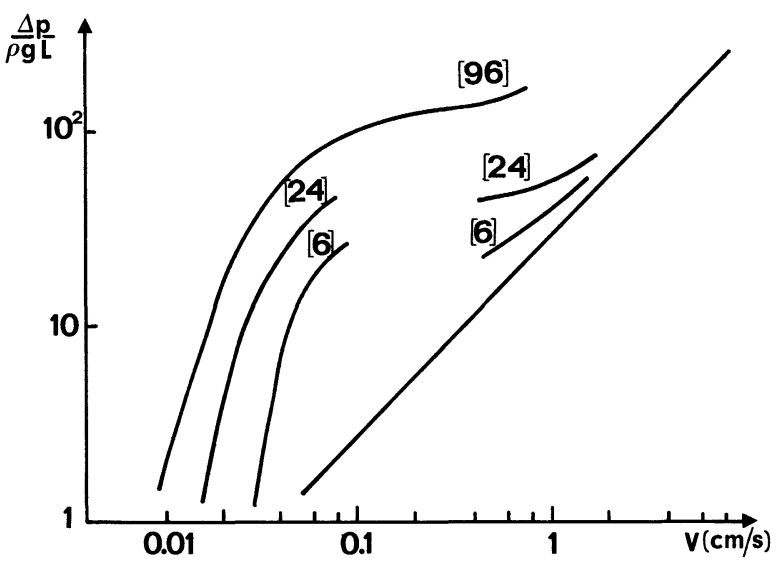

Fig. 1. - Courbes expérimentales de la perte de charge par unité de longueur : $\Delta p / \rho g L$ en fonction de la vitesse de débit $V$. La droite correspond au solvant. Les concentrations en p.p.m. sont indiquées entre crochets. [D'après [1].]

Nous allons montrer ici que toutes ces propriétés sont qualitativement expliquées par un modèle simple de transition coil-stretch [2] : en présence de gradients de vitesse longitudinaux $S$ alternatifs [3], la molécule est alternativement étirée et aplatie dans le sens de l'écoulement moyen.

En adoptant le modèle géométrique d'écoulement proposé dans la référence [1] (l'espace intersticiel est schématisé par un tube de section régulièrement variable), on peut estimer que le gradient de vitesse $S$ demeure égal à sa valeur moyenne $(S \sim 150 \mathrm{~V} / \mathrm{D}$, d'après [1]) avec un signe + ou - suivant que la 
section diminue ou augmente. La période peut être évaluée à $2 \theta \sim 0,2 D / V$, à partir du calcul de la vitesse moyenne effective. Ainsi, le produit $S \theta$ est constant et de l'ordre de $15 \pm 5$.

Ecrivons le bilan d'énergie par unité de volume et par unité de temps :

$$
V \frac{\Delta p}{L}=\eta_{0} S^{2}+n\langle\sigma\rangle .
$$

$\mathrm{Au}$ second membre, nous avons la dissipation visqueuse de la solution qui comporte deux termes : la dissipation newtonienne du solvant de viscosité $\eta_{0}$ et la dissipation due aux $n$ macromolécules par unité de volume. L'analyse de la référence [3] montre que $\langle\sigma\rangle$ peut être mis sous la forme :

$$
\langle\sigma\rangle=3 k_{\mathrm{B}} T \frac{1}{\tau_{0}} f\left(\frac{r_{\max }}{r_{0}}, S \tau_{0}\right)
$$

avec $k_{\mathrm{B}}$ : la constante de Boltzmann, $T$ : la température absolue, $\tau_{0}$ : le premier temps de relaxation moléculaire de Zimm et $r$ : l'élongation de la molécule $\left(r_{0}\right.$ : valeur au repos, $r_{\max }$ : valeur maximum). $f$ est une fonction sans dimension qui a été calculée pour un modèle particulier dans la référence [3].

Les principaux régimes sont alors les suivants :

1) Pour $S \lesssim 1 / \tau_{0}, x=r / r_{0}$ est petit, de l'ordre de $S \tau_{0}$, et la dissipation due aux macromolécules est $n\langle\sigma\rangle \sim\left(n r_{0}^{3}\right)\left(\eta_{0} S^{2}\right)$. Ceci correspond au régime newtonien avec la correction d'Einstein sur la viscosité.

2) Pour $S \gtrsim 1 / \tau_{0}, x$ devient rapidement grand (transition coil stretch) et $\langle\sigma\rangle$ croît en conséquence.

3) Pour $S \gg 1 / \tau_{0}$, on atteint un régime asymptotique simple que nous appellerons régime de déformation affine : la molécule se déforme exactement comme un élément de fluide. Le rapport $r_{\max } / r_{0}$ est alors entièrement déterminé par la géométrie de l'écoulement : il devient indépendant de $S$ ou de $V$. Dans cette limite, $\langle\sigma\rangle$ tend vers une constante, et les corrections par rapport au régime newtonien décroissent comme $1 / S$. En revenant à l'expression du gradient de pression, nous avons en effet :

$$
D \frac{\Delta p}{L} \simeq 10^{2}\left(\eta_{0} S+n \frac{\langle\sigma\rangle}{S}\right) .
$$

Ainsi, on obtient bien les traits principaux des courbes expérimentales. Plus précisément, nous avons

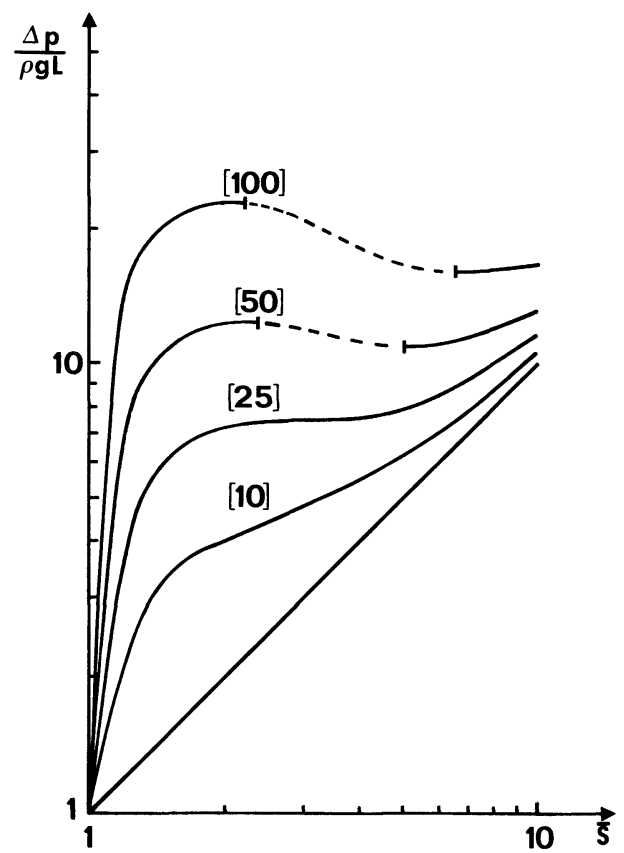

Fig. 2. - Courbes calculées de la perte de charge par unité de longueur : $\Delta p / \rho g L$ en fonction du gradient de vitesse réduit $\bar{S}=S \tau_{0}$. La droite correspond au solvant. Les concentrations en p.p.m. sont indiquées entre crochets. Les parties en pointillé ont des pentes négatives et correspondent à des régimes instables.

calculé $\langle\sigma\rangle$ en fonction de $S$ pour $S \theta=20$ et nous avons tracé sur la figure 2 des courbes de la perte de charge par unité de longueur $\Delta p / \rho g L$ en fonction du gradient de vitesse réduit $S \tau_{0}$ qui est proportionnel à la vitesse de débit $V$, et cela pour quelques valeurs de $c$ (compte tenu des valeurs numériques de la référence [1] : $\eta_{0}=10^{-2}$ poise, $M=10^{7}, \tau_{0}=6 \times 10^{-3} \mathrm{~s}$ et $D=2,2 \times 10^{-2} \mathrm{~cm}$ ). On relève sur cette figure les 3 propriétés annoncées. En outre, à partir d'une certaine concentration $(\sim 25$ p.p.m.), les courbes présentent une région de pente négative (en pointillé sur la figure) correspondant à un régime instable.

Les aspects les plus importants de la figure 1 sont ainsi retrouvés, mais nous dèvons souligner que l'accord n'est que qualitatif : 1) l'amplitude de l'écart calculé entre solution et solvant est plus faible que ce qui est effectivement observé, 2) l'instabilité n'apparaît qu'à partir d'une concentration de 25 p.p.m., mais ceci résulte de la faiblesse de l'écart relatif (c'est le produit $n\langle\sigma\rangle$ qui intervient), 3) la solution de concentration égale à 96 p.p.m. ne présente pas d'instabilité (Fig. 1). Ce dernier point peut sans doute être attribué aux enchevêtrements entre chaînes de polymère.

\section{Bibliographie}

[1] JAMEs, D. F. et McĹaren, D. R., J. Fluid Mech. 70 (1975) 733.

[2] De Gennes, P. G., J. Chem. Phys. 60 (1974) 5030.

[3] Daoudi, S., J. Physique 36 (1975) 1285. 